\title{
Comparison of Infrared Multiphoton Dissociation and Collision-Induced Dissociation of Supercharged Peptides in Ion Traps
}

\author{
James A. Madsen and Jennifer S. Brodbelt \\ Department of Chemistry and Biochemistry, The University of Texas at Austin, Austin, Texas, USA
}

\begin{abstract}
The number and types of diagnostic ions obtained by infrared multiphoton dissociation (IRMPD) and collision-induced dissociation (CID) were evaluated for supercharged peptide ions created by electrospray ionization of solutions spiked with $m$-nitrobenzyl alcohol. IRMPD of supercharged peptide ions increased the sequence coverage compared with that obtained by CID for all charge states investigated. The number of diagnostic ions increased with the charge state for IRMPD; however, this trend was not consistent for CID because the supercharged ions did not always yield the greatest number of diagnostic ions. Significantly different fragmentation pathways were observed for the different charge states upon CID or IRMPD with the latter yielding far more immonium ions and often fewer uninformative ammonia, water, and phosphoric acid neutral losses. Pulsed-Q dissociation resulted in an increase in the number of internal product ions, a decrease in sequence-informative ions, and reduced overall ion abundances. The enhanced sequence coverage afforded by IRMPD of supercharged ions was demonstrated for a variety of model peptides, as well as for a tryptic digest of cytochrome c. (J Am Soc Mass Spectrom 2009, 20, 349-358) (C) 2009 Published by Elsevier Inc. on behalf of American Society for Mass Spectrometry
\end{abstract}

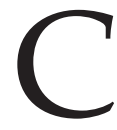
ollision-induced dissociation (CID) is the most common method used for sequencing peptides and identifying post-translational modifications (PTMs) [1], but uninformative labile neutral losses for both unmodified and modified peptides remain a consistent problem. Recently, electron capture dissociation (ECD) [2] in Fourier transform ion cyclotron resonance (FT-ICR) instruments, a method that involves the reaction of multiply charged cations with low-energy electrons, and electron-transfer dissociation (ETD) [3] in ion trap instruments, a technique that exploits the reaction of multiply charged cations with radical anions, have been developed to combat this neutral loss problem by affording complementary $c$ - and $z$-type backbone cleavages that leave labile modifications intact. These methods have been particularly promising for characterization of PTMs [4-8]; however, both techniques suffer from low fragmentation efficiencies, especially for lower charge states $[9,10]$, compared with CID.

Infrared multiphoton dissociation (IRMPD) is another tandem MS (MS/MS) method that has been successfully implemented in FT-ICR, time-of-flight, and quadrupole ion trap instrument systems [6, 11-24]. Much of the appeal of IRMPD in quadrupole ion traps

Address reprint requests to Dr. Jennifer S. Brodbelt, The University of Texas at Austin, Department of Chemistry and Biochemistry, 1 University Station A5300, Austin, TX 78712-0165. E-mail: jbrodbelt@mail.utexas.edu is related to the broader $\mathrm{m} / \mathrm{z}$ trapping range possible than that for conventional CID. This stems from the ability to reduce the radio frequency (rf) trapping voltage during ion activation, an option that is detrimental for CID as a result of the decrease in energy deposition associated with lower $\mathrm{rf}$ trapping voltages [25]. This low-mass cutoff (LMCO) problem associated with CID prohibits the detection of many diagnostic $b$ and $y$ ions of lower $\mathrm{m} / \mathrm{z}$ as well as immonium ions, the latter of which are particularly useful in determining the amino acid composition of unknown peptides. Furthermore, these lower $\mathrm{m} / \mathrm{z}$ diagnostic ions are important for de novo sequencing algorithms and can be useful for the rapid determination of modified amino acids (i.e., phosphorylated, acetylated, and methylated residues) [26-28]. Because all ions are continuously irradiated during the activation period, IRMPD can also promote secondary dissociation of primary product ions and thus lead to formation of a more diverse array of diagnostic ions compared with CID [25, 29]. Several methods have been explored to combat the low IRMPD efficiencies of larger peptides, including reducing the pressure and/or raising the temperature of the ion trap [28]. For example, the Glish group developed thermally assisted IRMPD (TA-IRMPD) [25], which involved heating the bath gas in the ion trap to increase the internal energies of ions. Our group has pursued peptide derivatization strategies to increase the photoab-
(C) 2009 Published by Elsevier Inc. on behalf of American Society for Mass Spectrometry. $1044-0305 / 09 / \$ 32.00$

doi:10.1016/j.jasms.2008.10.018
Published online November 5, 2008 Received July 25, 2008 Revised October 20, 2008 Accepted October 20, 2008 
sorptivity of ions and/or decrease their critical energies for dissociation [30-33].

Charge state has a significant impact on ion peptide dissociation because of changes in peptide conformation and proton mobility, with more highly charged states often yielding a greater array of structurally informative backbone cleavages [9, 34]. More highly charged peptides also have increased coulombic repulsion, which may facilitate dissociation compared with lower-charged species. Iavarone and Williams [35-39] explored the use of additives, such as $m$-nitrobenzyl alcohol ( $m$-NBA), that increase the surface tension and reduce the vapor pressure of electrospray ionization (ESI) droplets, thus generating higher protein and peptide charge states. This phenomenon has been termed supercharging. MS/MS has recently been used for the analysis of these supercharged ions, revealing interesting fragmentation trends such as enhanced formation of diagnostic ions upon CID of the highest supercharged state of proteins [38] and increased sequence coverage upon ETD of the highest supercharged state of tryptic peptides [9]. Although targeting ions in higher charge states can potentially increase the number of redundant product ions (i.e., those formed in multiple charges states), this has not proven to be a pervasive problem.

In the present study, the IRMPD and CID spectra of supercharged peptides obtained in ion trap mass spectrometers are compared. The more highly charged ions have lower critical energies; however, increased sequence coverage is most notable for IRMPD, which is attributed in part to the ongoing IR absorption and secondary dissociation of primary sequence ions throughout the activation period. Moreover, IRMPD allows detection of a greater array of diagnostic low $\mathrm{m} / \mathrm{z}$ ions. Activation of higher charge states is particularly advantageous for IRMPD since collisional cooling is competitive with energization attributed to the very low energy deposition per IR photon [40]. A comparison of pulsed-Q dissociation (P-QD) to IRMPD and CID is also assessed in this study.

\section{Experimental}

\section{Peptides, Proteins, and Reagents}

All peptides, proteins, and reagents were purchased from Sigma-Aldrich (St. Louis, MO, USA) except for the following: the peptides DRVYIHPFHLVIH and DAEFRHDSGYEVHHEK were purchased from Bachem Bioscience (King of Prussia, PA, USA), the phosphorylated peptides KRpTIRR and NRVpYIHPF from AnaSpec (San Jose, CA, USA), and immobilized TPCK trypsin beads were purchased from Pierce Biotechnology (Rockford, IL, USA).

\section{ESI-MS/MS}

For conventional analysis, model peptides were diluted to about $10 \mu \mathrm{M}$ with $49.5 \% / 49.5 \% / 1.0 \%$ water/metha- nol/acetic acid solution before direct infusion at 3 $\mu \mathrm{L} / \mathrm{min}$ into the mass spectrometer. Peptides were supercharged by the addition of $1-2 \%$ (vol/vol) $m$-NBA into the above-cited working solutions before MS analysis.

Model peptides were analyzed using a modified Thermo Fisher (San Jose, CA, USA) LTQ two-dimensional linear ion trap mass spectrometer equipped with a Synrad 50-W continuous-wave $\mathrm{CO}_{2}$ laser (Model 48-5; Mukilteo, WA, USA). Laser radiation at a wavelength of $10.6 \mu \mathrm{m}$ was introduced into the linear ion trap via a modified back plate containing a ZnSe window [33]. Tryptic peptides were analyzed using a modified ThermoFinnigan LCQ Deca XP three-dimensional quadrupole ion trap mass spectrometer equipped with the same Synrad 50-W continuous-wave $\mathrm{CO}_{2}$ laser [30]. Radiation at $10.6 \mu \mathrm{m}$ was introduced into the quadrupole ion trap through a 5-mm hole drilled into the ring electrode. With the laser operated at full power, irradiation times of 10-250 ms (LTQ) and 25-500 ms (LCQ) were used with an activation $q$ value of 0.1 during IRMPD experiments. For certain tryptic peptides, the He bath gas pressure was reduced during laser irradiation to allow for greater fragmentation efficiencies. A higher $q$ value of 0.25 was used for CID experiments to afford more efficient ion activation at the expense of a more limited $m / z$ trapping range. For all charge states, the CID voltage or IR irradiation time was adjusted to cause dissociation of about $100 \%$ (if possible) of the precursor ions. P-QD experiments were performed on the LTQ mass spectrometer with a $q$ value of 0.7 and an activation time of $0.1 \mathrm{~ms}$. For the present study, a product ion is considered to be detected in a CID, P-QD, or IRMPD mass spectrum if the ion peak has a signalto-noise ratio $(S / N) \geq 3$. Dissociation efficiencies were calculated based on the percentage of precursor ion abundance (based on ion peak area) converted into product ions, where a value of $100 \%$ signifies that the initial abundance of the isolated precursor ion is completely accounted for by the summed abundances of all product ions. Sequencing efficiencies (SEFF) were calculated based on the percentage of precursor ion abundance (based on ion peak area) converted into diagnostic ions (e.g., $b, y$, and immonium). A value of $100 \%$ indicates that the entire abundance of the selected precursor ion is converted into sequence-informative product ions. Although neutral losses, such as loss of ammonia or water, can give some useful information pertaining to the sequence of a peptide, these ions are often redundant or complicate spectra and are thus not classified as informative-sequence ions. SEFF is a more useful parameter for assessing the diagnostic value of an MS/MS experiment, since product ions produced from neutral losses, such as ammonia loss or via dehydration, and internal fragments are not included. Moreover, redundant multiply charged product ions were not counted in total diagnostic ion counts nor included in SEFF calculations. For the objectives of the present study, the term "immonium ion" is used to encompass both immonium and immonium-related ions. 


\section{Tryptic Digest}

Cytochrome c $(50 \mu \mathrm{M})$ was digested with $100 \mu \mathrm{L}$ immobilized TPCK trypsin beads in $50 \mathrm{mM}$ ammonium bicarbonate incubated for $18 \mathrm{~h}$ at $37^{\circ} \mathrm{C}$. After digestion, the excess beads were discarded and the remaining tryptic peptides were diluted to $10 \mu \mathrm{M}$ with $49.5 \%$ / $49.5 \% / 1.0 \%$ water/methanol/acetic acid solution before analysis. The tryptic peptides were supercharged by the addition of $1-2 \% m-\mathrm{NBA}$. Working solutions were directly infused into the mass spectrometer at $3 \mu \mathrm{L} / \mathrm{min}$.

\section{Results and Discussion}

IRMPD experiments were undertaken by using both a two-dimensional linear ion trap mass spectrometer and a three-dimensional quadrupole ion trap mass spectrometer. IRMPD yielded the same number of diagnostic ions for both instruments; however, the irradiation times were approximately four times lower for the linear ion trap. The improved dissociation efficiency for the linear ion trap is attributed to a longer laser path length and/or better overlap of the laser with the ion cloud. For a more systematic investigation, the linear ion trap was used for all CID and IRMPD comparisons of model peptides and the quadrupole ion trap was used for all CID and IRMPD comparisons of tryptic peptides.

\section{Model Peptides}

The addition of $m$-NBA to the peptide solutions typically increased the maximum observed charge state by one through the addition of one extra proton with little overall impact on total ion abundances. For example, by conventional ESI-MS analysis the peptide KFHEKHHSHRGY yielded a highest charge state of $4+$ as seen in Figure 1a. However, with the addition of $m$-NBA the $5+$ supercharged state emerged as the dominant ion (Figure 1b). All four observed charge states of the peptide were subjected to IRMPD, as illustrated in Figure $1 c-f$. The IRMPD spectrum of the $2+$ charge state is dominated by the intact precursor ion and only a few product ions of low abundance. The triply protonated peptide also exhibits relatively low IR photodissociation efficiency (i.e., only $52 \%$ of the original isolated precursor ion is converted into product ions), with much of the precursor ion surviving the irradiation period and only a modest number of diagnostic ions formed. Exposure of the $2+$ and $3+$ ions to long irradiation times $(250 \mathrm{~ms})$ did not yield a high degree of product ions, a factor attributed to the competition between IR energization and collisional cooling. However, the higher charge states (e.g., $4+$ and 5+) required shorter irradiation times (100 ms), displayed much higher IRMPD efficiencies (up to $75 \%$ conversion of parent ions into total product ions), and yielded greater numbers of diagnostic ions. The sequencing efficiency (SEFF) ranged from $8 \%$ for the doubly charged peptide up to $39 \%$ for the highest charge state $(5+)$. Activation of the highest charge state by
IRMPD resulted in the greatest proportional yield of sequence-informative ions ( $b, y$, and immonium). The $5+$ supercharged state of KFHEKHHSHRGY also produced the most extensive sequence coverage upon IRMPD with $6 b$ ions, $10 y$ ions, and 3 immonium ions. For CID of this same peptide (Figure 2), all charge states yielded a similar number of sequence ions (on average $4 b$ ions and $7 y$ ions). The $3+$ charge state yielded the greatest number of diagnostic ions upon CID, yet produced only 13 total sequence ions ( $6 b$ ions and $7 y$ ions) and no immonium ions. High CID dissociation efficiencies were achieved for all charge states, ranging from 68 to $87 \%$ conversion of the selected precursor ions into product ions. Importantly, SEFF values ranged from $16 \%$ for the $4+$ charge state up to $36 \%$ for both the $3+$ and $5+$ charge states, all of which were lower than the supercharged state $(5+)$ dissociated by IRMPD.

The supercharged states also yielded greater sequence coverage for peptides that readily absorb IR radiation, such as phosphorylated peptides. As seen for the series of IRMPD spectra of the phosphorylated peptide KRpTIRR in Figure 3, the loss of phosphoric acid dominates the spectra for the lower charge states $(1+$ to $3+)$. In contrast, the greatest array of diagnostic ions is seen for the $4+$ peptide by IRMPD. Figure 3 illustrates that the total sequence coverage increases with each additional charge. Phosphoric acid loss is significant upon IRMPD of the lower charge states but has less of an impact for the higher charge states and is still less substantial than that observed upon CID.

A series of 8 peptides were analyzed by both IRMPD and CID to explore the impact of supercharging on the sequence coverage for peptides as a function of their size (6 to 16 amino acids) and phosphorylation status. The results are summarized in bar graph form in Figure 4. For peptides subjected to IRMPD as seen in Figure 4a, the number of diagnostic ions increased with increasing charge state, with the supercharged state always producing the greatest number of informative ions. For CID (Figure 4b), however, this trend was not consistent and the supercharged ion did not always yield the greatest number of diagnostic ions. Activation of the higher charged ions often required lower CID voltages than lower charge states. Those peptides for which the higher charge states produced fewer diagnostic ions upon CID typically underwent dehydration, which accounts for their less diagnostic fragmentation patterns. For IRMPD, however, fewer of these uninformative neutral losses were seen for the supercharged states, which may be in part the result of rapid secondary dissociation of these primary product ions into more useful diagnostic ions. This phenomenon could explain the general enhancement in the total number of informative product ions observed for IRMPD compared with CID.

Figure $4 c$ shows a side-by-side comparison of CID and IRMPD for the best-performing charge states (i.e., the charge state that produced the greatest total number 
(a)

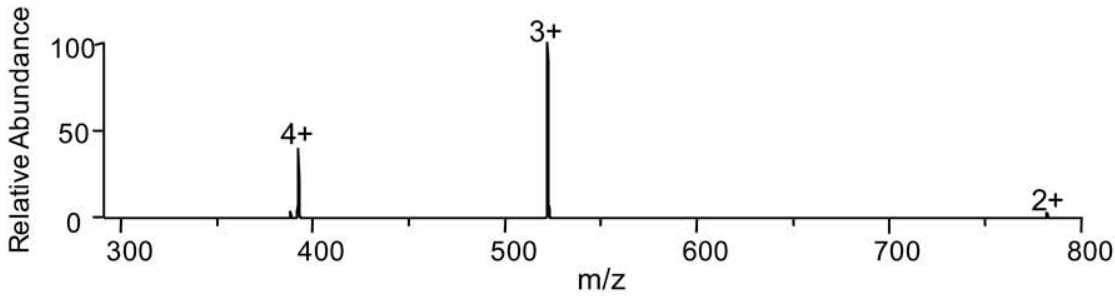

(b)
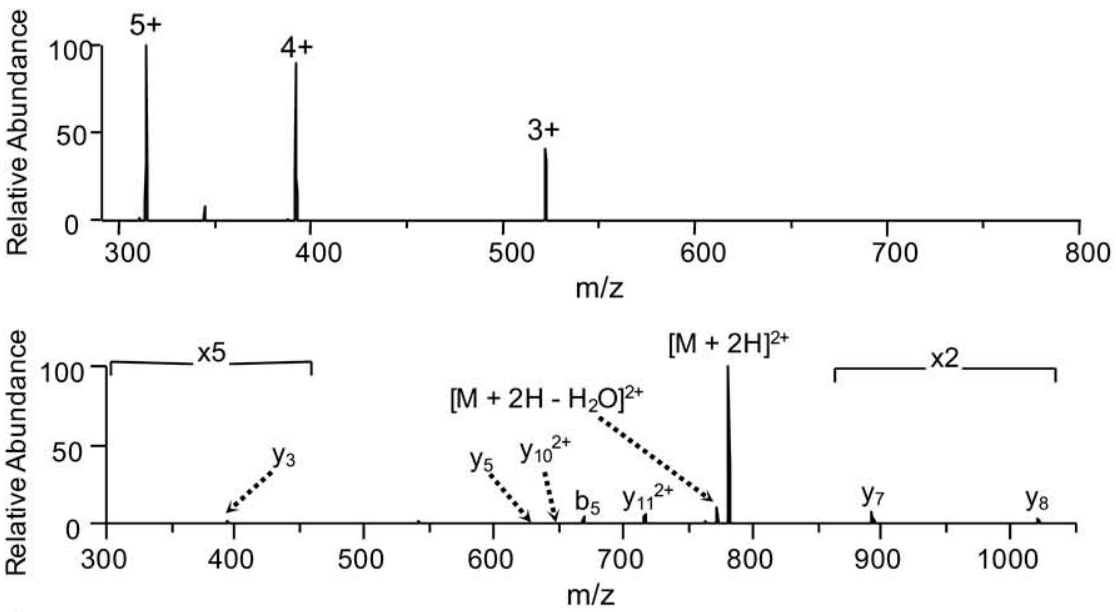

(c)

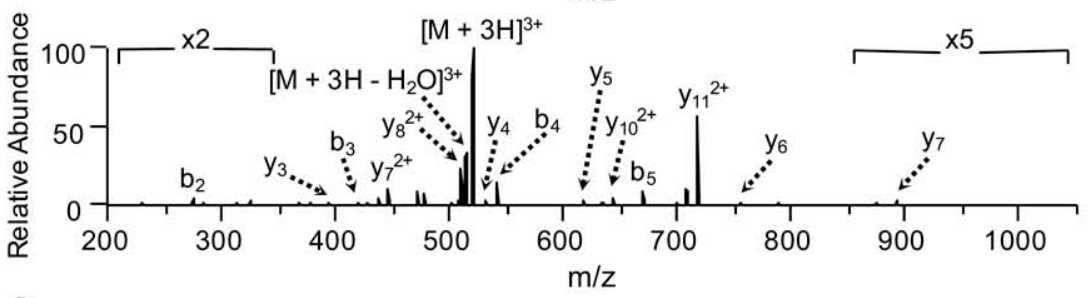

(d)

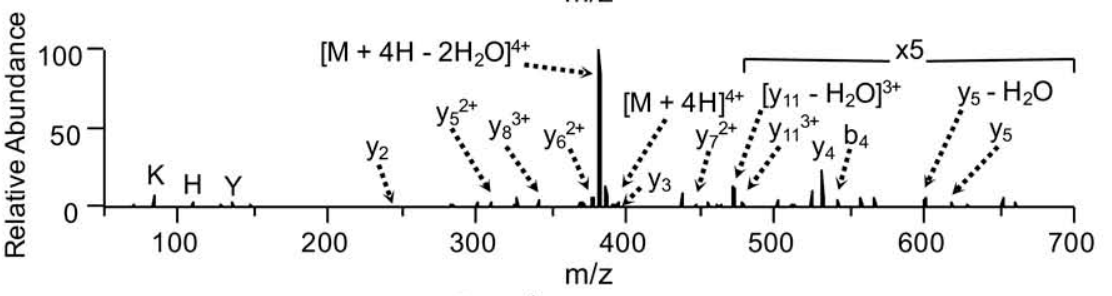

(e)

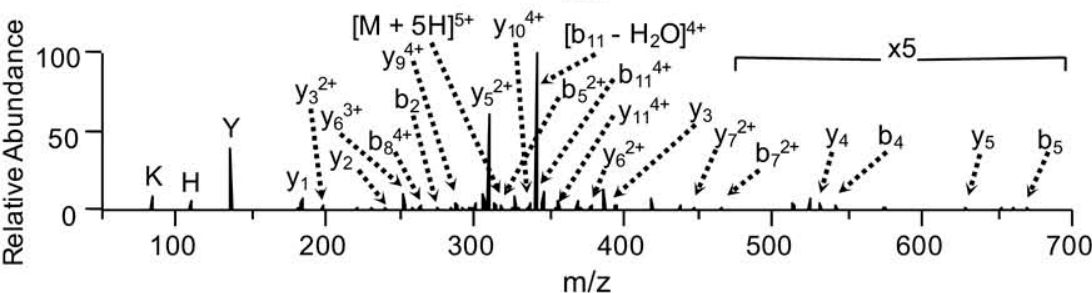

Figure 1. ESI mass spectra of KFHEKHHSHRGY (a) with $1 \%$ acetic acid and $49.5 \% / 49.5 \%$ $\mathrm{MeOH} / \mathrm{H}_{2} \mathrm{O}$, (b) supercharged with $1 \% \mathrm{~m}$-NBA, $1 \%$ acetic acid, and $49 \% / 49 \% \mathrm{MeOH} / \mathrm{H}_{2} \mathrm{O}$. IRMPD mass spectra of the protonated peptide KFHEKHHSHRGY (c) $2+$ charge state, $50 \mathrm{~W}, \mathrm{t}=250 \mathrm{~ms}$; (d) $3+$ charge state, $50 \mathrm{~W}, \mathrm{t}=250 \mathrm{~ms} ;(\mathbf{e}) 4+$ charge state, $50 \mathrm{~W}, \mathrm{t}=100 \mathrm{~ms}$; and (f) $5+$ supercharged state, $50 \mathrm{~W}, \mathrm{t}=100 \mathrm{~ms}$.

of diagnostic ions) for each model peptide included in the study. This bar graph comparison confirms that IRMPD of the supercharged peptide uniformly affords the greatest sequence coverage. This increased sequence coverage for IRMPD is attributed to a combination of three factors: (1) reduction in the ion critical energies for higher charge states arising from some combination of coulombic effects and enhanced proton mobility, (2) broader $\mathrm{m} / \mathrm{z}$ trapping range attributed to alleviation of the low-mass cutoff, and (3) ongoing IR absorption and secondary dissociation that leads to a greater array of ions and transformation of less informative ions (i.e., product ions from neutral loss of water, ammonia, or phosphoric acid) into more diagnostic ones. 
(a)

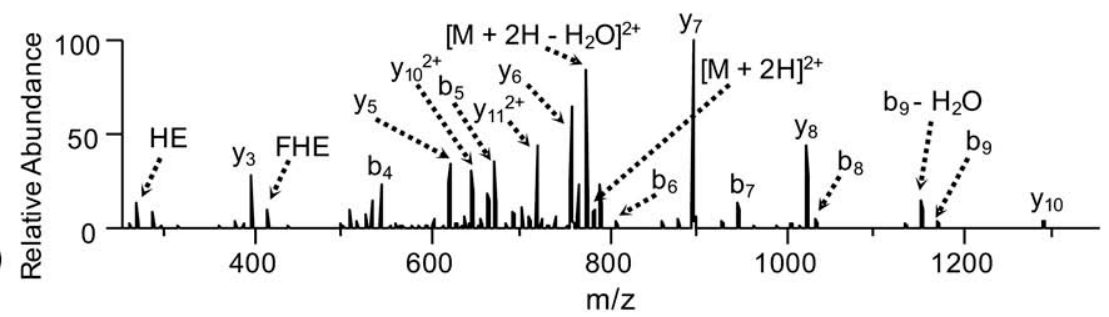

(b)

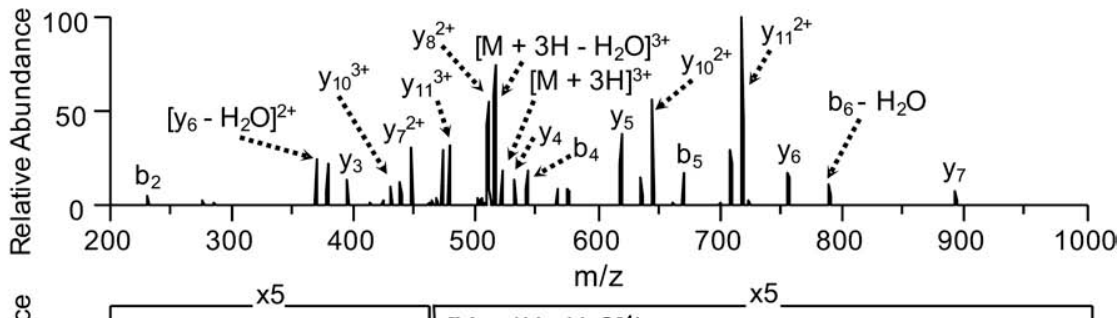

(c)

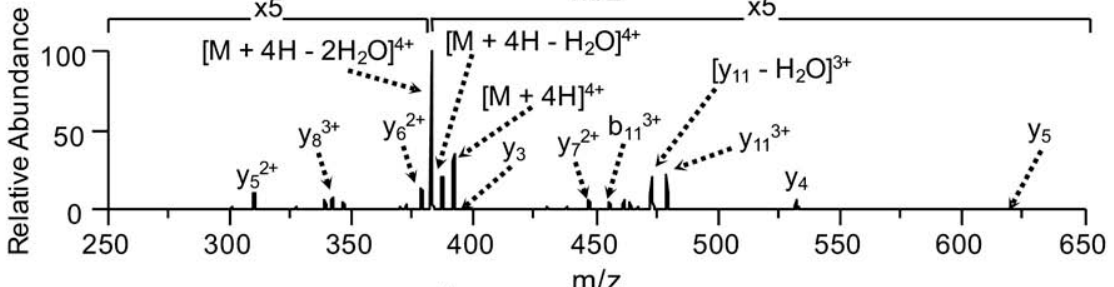

(d)

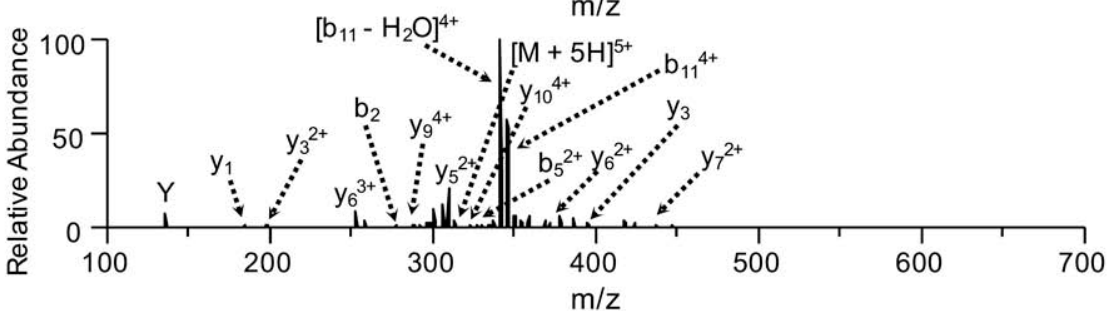

Figure 2. CID mass spectra of the protonated peptide KFHEKHHSHRGY (a) $2+$ charge state, $29 \mathrm{mV}$ collision voltage; (b) $3+$ charge state, $24 \mathrm{mV}$ collision voltage; (c) $4+$ charge state, $17 \mathrm{mV}$ collision voltage; and (d) $5+$ supercharged state, $16 \mathrm{mV}$ collision voltage.

\section{Tryptic Digest of Cytochrome $\mathrm{c}$}

In a manner similar to that described earlier for the model peptides, IRMPD and CID were used to evaluate a series of tryptic peptides from cytochrome c. The tryptic digest was mixed with $1 \% m$-NBA, and then each charge state of each tryptic peptide was analyzed by CID and IRMPD. The number of diagnostic product ions $(b, y$, and immonium ions) were tabulated and the results are summarized in Figure 5a and b, respectively. An increasing number of diagnostic ions were formed for higher peptide charge states by IRMPD of the tryptic peptides, a trend not observed for CID. Also, IRMPD of the supercharged ions consistently yielded a greater number of diagnostic ions compared with CID. As confirmed again in a side-by-side bar graph that highlights the best CID results compared with the best IRMPD results for the series of tryptic peptides, IRMPD of each supercharged peptide afforded the greatest number of diagnostic ions in every case (Figure 5c). $m$-NBA has been used previously to create supercharged ions of tryptic peptides analyzed by LC-MS [9] and we expect that this strategy combined with IRMPD would further facilitate protein characterization.
The dissociation efficiencies for conversion of precursor ions into product ions were also assessed for various tryptic peptides and charge states. For example, for the $1+$ charge states of EDLIAYLK and GITWK, only 26 and $18 \%$ of the parent ions, respectively, were converted into product ions upon IR irradiation. Conversely, the IRMPD efficiencies increased to 43 and $48 \%$, respectively, for the $2+$ charge state. In comparison, the CID efficiencies ranged from 32 to $53 \%$ and 36 to $51 \%$ for the two charge states of EDLIAYLK and GITWK, respectively. For the peptide IFVQKCAQCHTVEK, the IRMPD efficiencies ranged from $55 \%$ for the $1+$ charge state to $61 \%$ for the doubly charged state to $57 \%$ for the triply charged state. In comparison, the CID efficiencies ranged from 47 to $61 \%$ for the same peptide. Similar IRMPD efficiencies and trends were seen for the $1+$, $2+$, and $3+$ charge states of TGPNLHGLFGR and the $2+$ and $3+$ charge states of HKTGPNLHGLFGRK.

SEFF values were also compared with the more traditional dissociation efficiencies for the tryptic peptides described in the previous paragraph. CID of EDLIAYLK and GITWK yielded SEFF values ranging from 6 to $5 \%$ and 5 to $10 \%$ for the $1+$ and $2+$ charge states, respectively. 

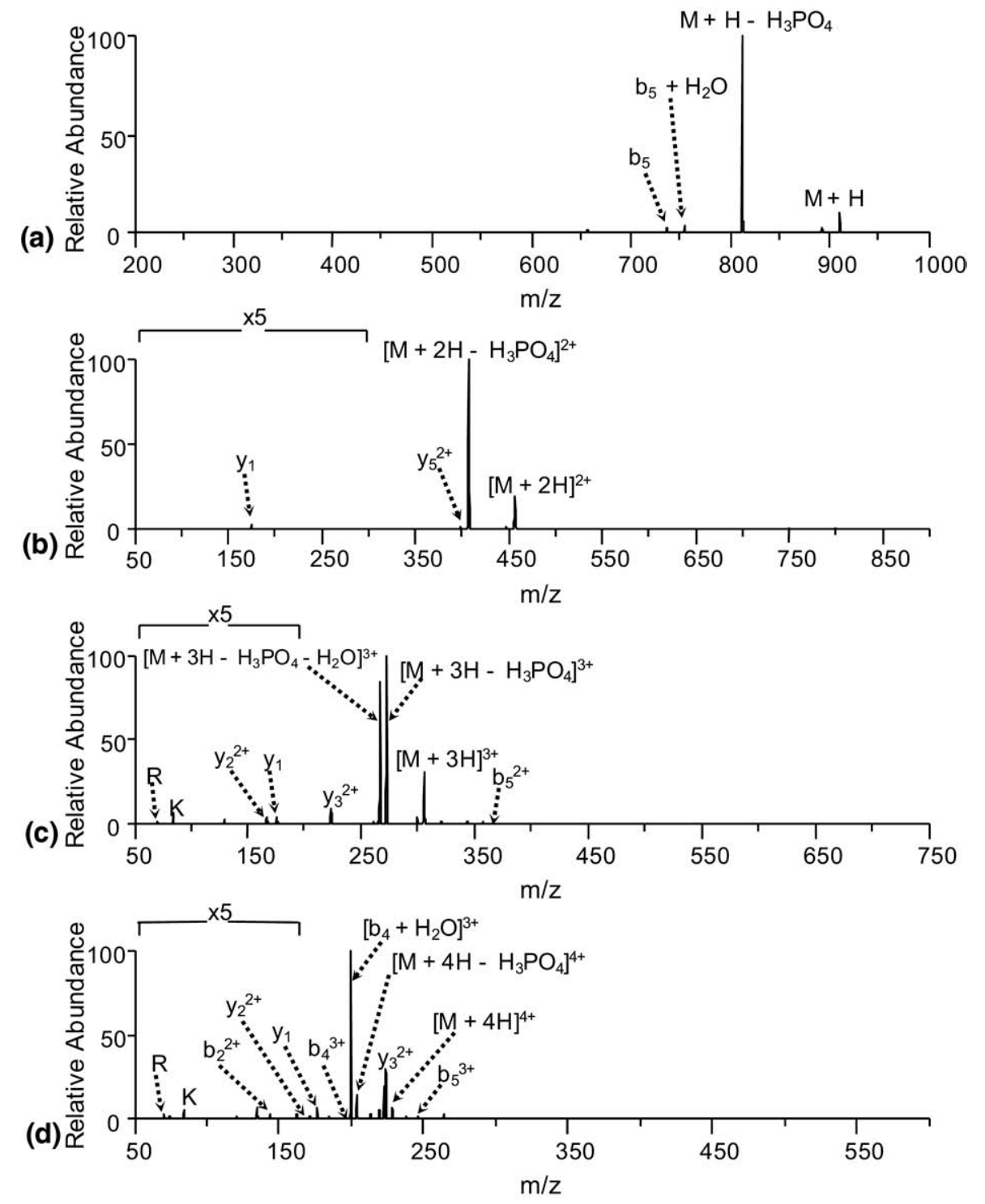

Figure 3. IRMPD mass spectra of the protonated phosphorylated peptide KRpTIRR, (a) $1+$ charge state, $50 \mathrm{~W}, \mathrm{t}=14 \mathrm{~ms}$; (b) $2+$ charge state, $50 \mathrm{~W}, \mathrm{t}=14 \mathrm{~ms}$; (c) $3+$ charge state, $50 \mathrm{~W}, \mathrm{t}=14 \mathrm{~ms}$; and (d) $4+$ supercharged state, $50 \mathrm{~W}, \mathrm{t}=10 \mathrm{~ms}$.

Conversely, SEFF values obtained by IRMPD for the same two peptides in the singly charged and doubly charged states ranged from 4 to $27 \%$ and 2 to $30 \%$, respectively. For the peptide IFVQKCAQCHTVEK, the IRMPD SEFF values ranged from $8 \%$ for the $1+$ charge state to $19 \%$ for the $2+$ charge state and up to $21 \%$ for the $3+$ charge state. In comparison, the CID SEFF values ranged from 11 to $19 \%$ for the same peptide. Thus, although IRMPD often resulted in lower dissociation efficiencies (i.e., percentage of original isolated precursor converted into total product ions) compared with CID, the SEFF values (original isolated precursor converted into diagnostic product ions) for the supercharged states were often higher for IRMPD. This increase in the SEFF values for IRMPD of the highest charge states reflects both the diminishment in uninformative neutral losses (e.g., $\mathrm{H}_{2} \mathrm{O}, \mathrm{NH}_{3}$ ) and reduction in the formation of internal ions compared with CID.
Figure 6 shows the trends in the distributions of $b, y$, and immonium ions for one representative tryptic peptide, HKTGPNLHGLFGRK, in a quadrupole ion trap obtained by CID as the CID voltage is varied or by IRMPD as the irradiation time is varied. Figure $6 a$ and $b$ show the distributions for the $3+$ charge state and the results for the $2+$ charge state are shown in Figure $6 \mathrm{c}$ and d. At longer irradiation times for the triply charged peptide, IRMPD yielded significantly different distributions of product ions, including immonium ions and more $b$ and $y$ ions, compared with CID. For IRMPD, higher irradiation times were needed to dissociate the doubly charged peptide and the sequence coverage was lower. For CID, the total number of sequence ions observed was the same for both charge states. On average, a total of $12 b$ and $y$ ions were seen for both charge states using CID, with one additional $b$ ion and one fewer $y$ ion detected for the $2+$ charge state, 
(a)
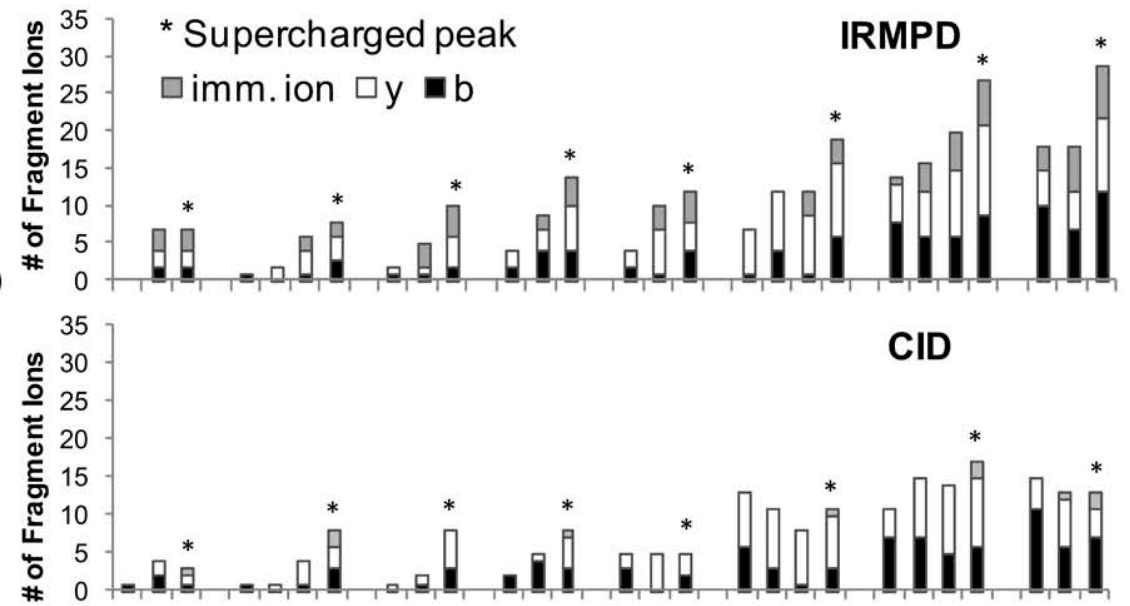

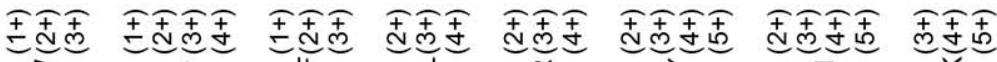

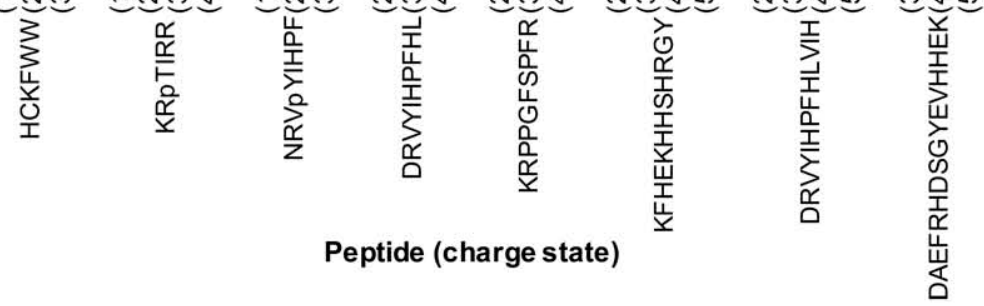

(b)

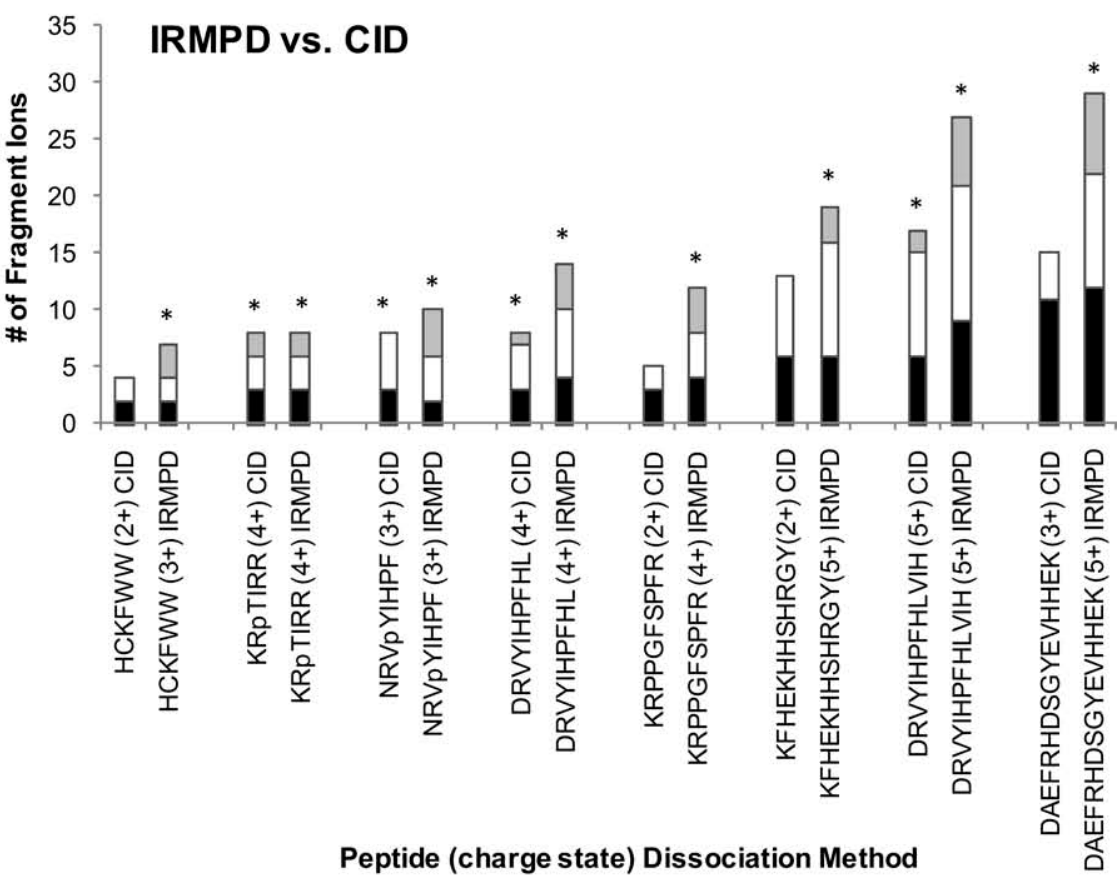

Figure 4. Number of diagnostic ions ( $y, b$, and immonium) from different charge states of protonated peptides (a) using IRMPD (b) and CID. The best performers (i.e., most diagnostic ions) for each method (e.g., IRMPD and CID) are compared in (c). The average IRMPD irradiation times were $98 \mathrm{~ms}$ $(1+), 116 \mathrm{~ms}(2+), 84 \mathrm{~ms}(3+), 51 \mathrm{~ms}(4+)$, and $48 \mathrm{~ms}(5+)$. Activation times for CID were $30 \mathrm{~ms}$ with average collisional voltages of $40 \mathrm{mV}(1+), 26 \mathrm{mV}(2+), 20 \mathrm{mV}(3+), 16 \mathrm{mV}(4+)$, and $14 \mathrm{mV}(5+)$.

compared with the triply charged species. For the $2+$ charge state, an average of $7 b, 2 y$, and 3 immonium ions were seen when using IRMPD. Conversely, the average total $b$ and $y$ ion count increased to 16 (7 $b$ and $9 y$ ions) and an average of 5 immonium ions were seen with IRMPD of the $3+$ charge state.

\section{Secondary Dissociation of Product Ions Using $M S^{3}$}

To further explore the occurrence of secondary dissociation during IRMPD, a product ion stemming from the neutral loss of water and another that incorporates the addition of water were selectively isolated and subjected 
(a)
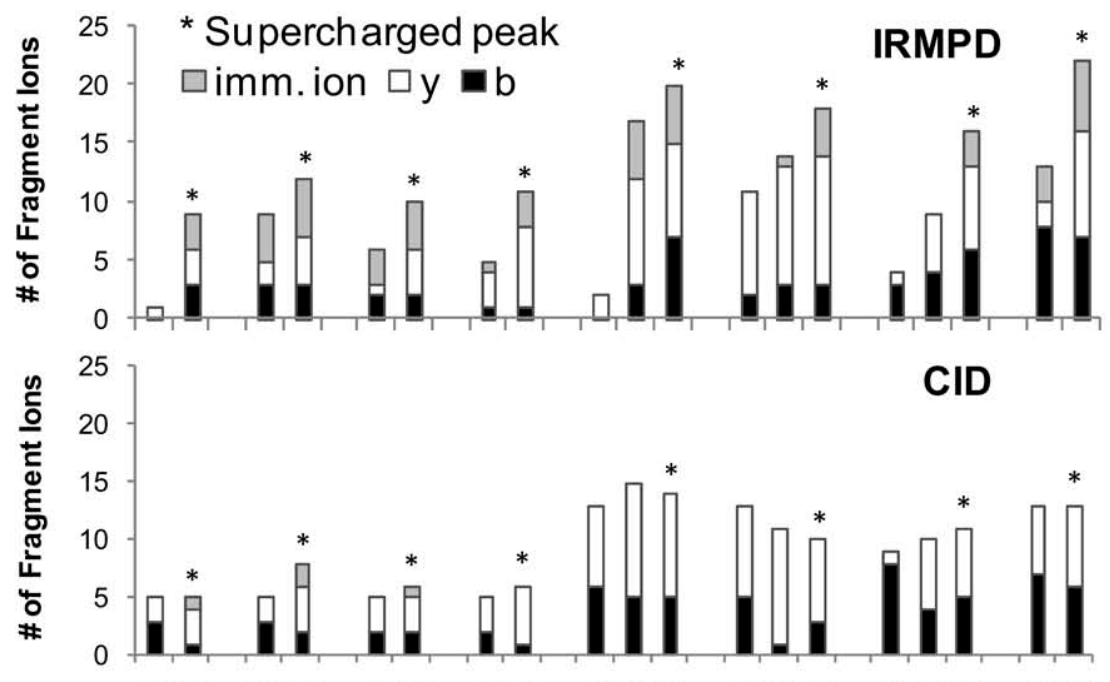

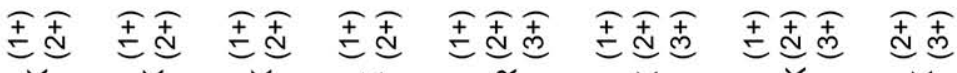

旁 冚

(b)

Peptide (charge state)
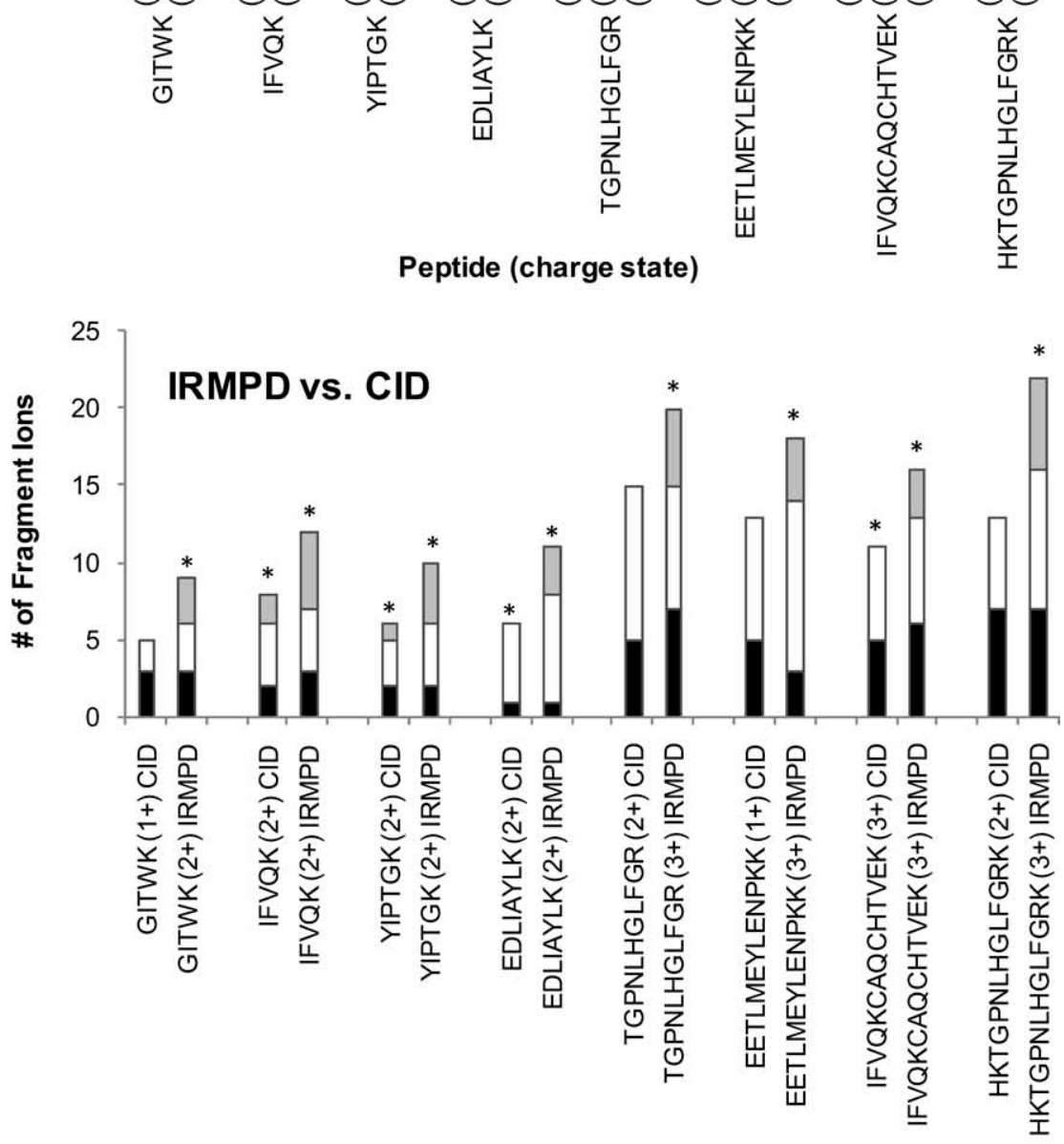

(c)

Peptide (charge state) Dissociation Method

Figure 5. Number of diagnostic ions $(y, b$, and immonium) of various tryptic peptides from cytochrome c (a) using IRMPD (b) and CID. The best performers (i.e., most diagnostic ions) for each method (e.g., IRMPD and CID) are compared in (c). The average IRMPD irradiation times were 209 $\mathrm{ms}(1+), 123 \mathrm{~ms}(2+)$, and $50 \mathrm{~ms}(3+)$. Activation times for CID were $30 \mathrm{~ms}$ with average collisional voltages of $0.93 \mathrm{~V}(1+), 0.53 \mathrm{~V}(2+)$, and $0.42 \mathrm{~V}(3+)$.

to a second stage of ion activation in $\mathrm{MS}^{3}$ experiments (Supplemental Figure 1, which can be found in the electronic version of this article). The $3+$ charge state of the peptide DRVYIHPFHL was collisionally activated to promote dehydration, resulting in the $\left[\mathrm{M}-\mathrm{H}_{2} \mathrm{O}\right]^{3+}$ ion of high abundance. This product ion was then isolated and 

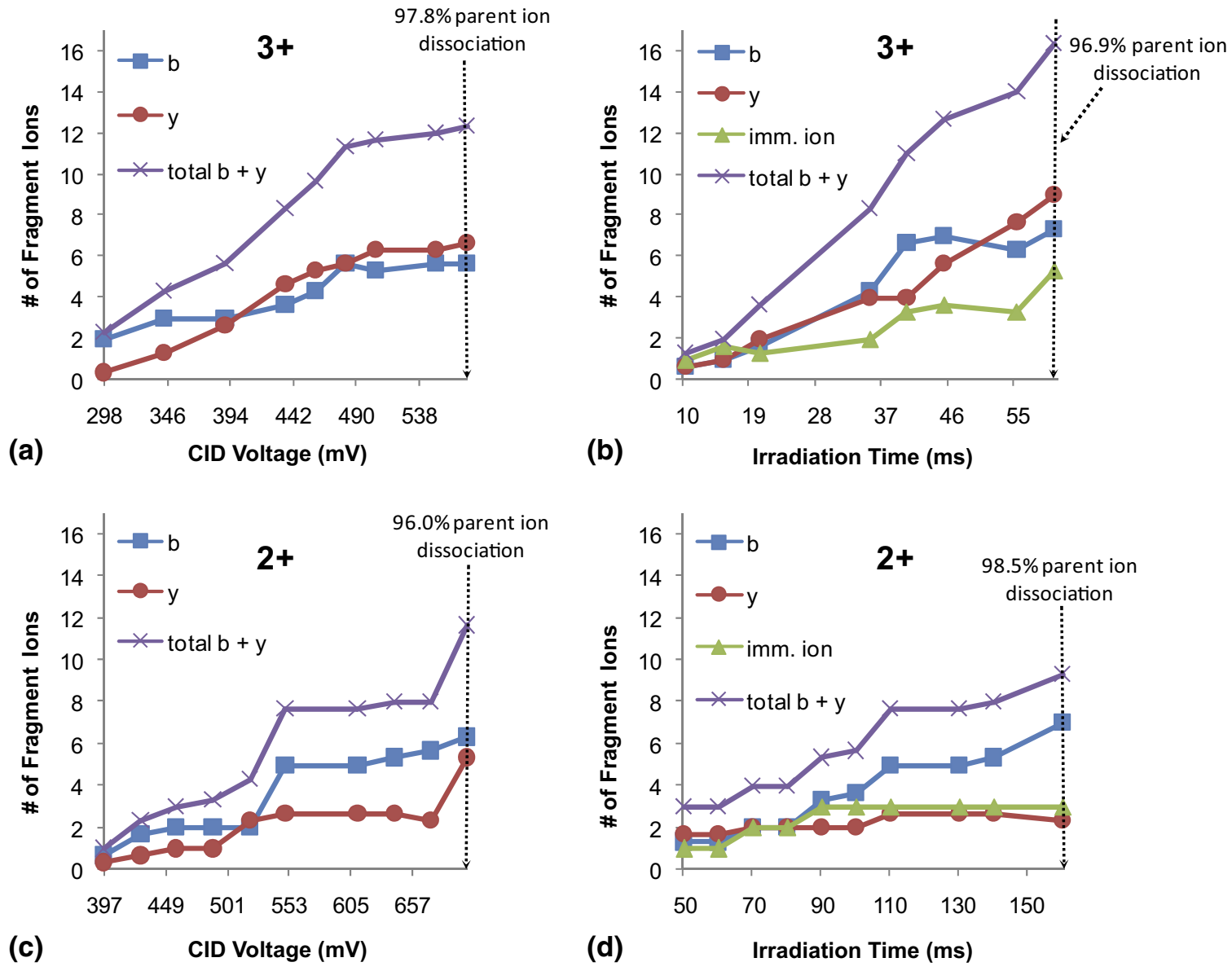

Figure 6. The distribution of diagnostic ions as a function of CID voltage or IRMPD irradiation time for protonated HKTGPNLHGLFGRK (3+) (a) CID, (b) IRMPD and HKTGPNLHGLFGRK (2+), (c) CID, and (d) IRMPD.

activated by IRMPD, thus resulting in the formation of two abundant $b$ sequence ions and one immonium ion but very few secondary ions attributed to uninformative losses of water or ammonia. The triply charged DRVYIHPFHL also produced a primary product ion, $\left[\mathrm{b}_{9}+\mathrm{H}_{2} \mathrm{O}\right]^{2+}$, of high abundance upon CID. This product ion was isolated and subjected to IRMPD, yielding three abundant $b$ sequence ions and one immonium ion as well as a few internal product ions of low abundance. These $\mathrm{MS}^{3}$ results suggest that the less informative neutral loss and neutral addition products ions that are commonly formed upon CID (or IRMPD) can be converted to more diagnostic product ions upon further activation. Since CID is a resonant process that causes energization of solely the selected precursor ion, it is only the nonresonant IRMPD method that can successfully convert the less informative primary product ions into more informative secondary product ions.

\section{$P-Q D$ Comparison}

As a final comparison, the tryptic peptide HKTGPNLHGLFGRK in the $3+$ charge state was subjected to P-QD [41], to assess the total number of diagnostic ions since this dissociation method, like IRMPD, alleviates the LMCO problem associated with CID. P-QD entails activating the precursor ion at a high $q$ value and high CID energy for a short time and then quickly lowering the trapping rf voltage to detect product ions of low $\mathrm{m} / \mathrm{z}$. At a P-QD voltage of $46 \mathrm{mV}$, only four diagnostic ions were observed, with much of the ion signal attributed to undesirable internal fragments at $m / z$ values that clutter the range where $b$ and $y$ ions would be found (Supplemental Figure 2). As the P-QD voltage was increased to $53 \mathrm{mV}$, the total ion abundance was reduced by $85 \%$, the level of noise increased, and a total of only two sequence ions were detected (spectrum not shown). Increasing the P-QD voltage beyond $66 \mathrm{mV}$ decreased the total ion abundance by over $99 \%$ and the noise level obscured any fragment ion peaks. For comparison, when this triply protonated peptide was subjected to IRMPD, 22 diagnostic ions were produced with a low abundance of uninformative internal fragment ions (Figure 5a).

\section{Conclusions}

IRMPD of the supercharged states of peptide ions resulted in the most informative array of sequence ions compared with dissociation of lower charge states by 
IRMPD or upon dissociation of any charge state by CID. In addition, the number of immonium ions was dramatically increased with IRMPD of supercharged ions, which could aid in identification of PTMs. In general, the total number of diagnostic ions increased with increasing charge state for IRMPD. This trend was not observed for CID, however, and often lower charge states led to the greatest number of diagnostic ions. IRMPD of supercharged ions is anticipated to be particularly beneficial for larger peptides or proteins to maximize sequence coverage.

\section{Acknowledgments}

We gratefully acknowledge funding from National Science Foundation Grant CHE-0718320 and Welch Foundation Grant F1155.

\section{References}

1. Wells, J. M.; McLuckey, S. A. Collision-Induced Dissociation (CID) of Peptides and Proteins. Methods Enzymol. 2005, 402, 148-185.

2. Zubarev, R. A.; Kelleher, N. L.; McLafferty, F. W. Electron Capture Dissociation of Multiply Charged Protein Cations. A Nonergodic Process. J. Am. Chem. Soc. 1998, 120, 3265-3266.

3. Syka, J. E. P.; Coon, J. J.; Schroeder, M. J.; Shabanowitz, J.; Hunt, D. F. Peptide and Protein Sequence Analysis by Electron Transfer Dissociation Mass Spectrometry. Proc. Natl. Acad. Sci. U. S. A. 2004, 101, $9528-$ 9533.

4. Mirgorodskaya, E.; Roepstorff, P.; Zubarev, R. A. Localization of O-Glycosylation Sites in Peptides by Electron Capture Dissociation in a Fourier Transform Mass Spectrometer. Anal. Chem. 1999, 71, 4431-4436.

5. Stensballe, A.; Jensen, O. N.; Olsen, J. V.; Haselmann, K. F.; Zubarev, R. A. Electron Capture Dissociation of Singly and Multiply Phosphorylated Peptides. Rapid Commun. Mass Spectrom. 2000, 14, 1793-1800.

6. Hakansson, K.; Cooper, H. J.; Emmett, M. R.; Costello, C. E.; Marshall, A. G.; Nilsson, C. L. Electron Capture Dissociation and Infrared Multiphoton Dissociation MS/MS of an N-Glycosylated Tryptic Peptide to Yield Complementary Sequence Information. Anal. Chem. 2001, $73,4530-4536$.

7. Kleinnijenhuis, A. J.; Kjeldsen, F.; Kallipolitis, B.; Haselmann, K. F.; Jensen, O. N. Analysis of Histidine Phosphorylation Using Tandem MS and Ion-Electron Reactions. Anal. Chem. 2007, 79, 7450-7456.

8. Srikanth, R.; Wilson, J.; Bridgewater, J. D.; Numbers, J. R.; Lim, J.; Olbris, M. R.; Kettani, A.; Vachet, R. W. Improved Sequencing of Oxidized Cysteine and Methionine Containing Peptides Using Electron Transfer Dissociation. J. Am. Soc. Mass Spectrom. 2007, 18, 1499-1506.

9. Kjeldsen, F.; Giessing, A. M. B.; Ingrell, C. R.; Jensen, O. N. Peptide Sequencing and Characterization of Post-Translational Modifications by Enhanced Ion-Charging and Liquid Chromatography Electron-Transfer Dissociation Tandem Mass Spectrometry. Anal. Chem. 2007, 79, 92439252 .

10. Swaney, D. L.; McAlister, G. C.; Wirtala, M.; Schwartz, J. C.; Syka, J. E. P.; Coon, J. J. Supplemental Activation Method for High-Efficiency Electron-Transfer Dissociation of Doubly Protonated Peptide Precursors. Anal. Chem. 2007, 79, 477-485.

11. Little, D. P.; Speir, J. P.; Senko, M. W.; O'Connor, P. B.; McLafferty, F. W. Infrared Multiphoton Dissociation of Large Multiply Charged Ions for Biomolecule Sequencing. Anal. Chem. 1994, 66, 2809-2815.

12. Li, W.; Hendrickson, C. L.; Emmett, M. R.; Marshall, A. G. Identification of Intact Proteins in Mixtures by Alternated Capillary Liquid Chromatography Electrospray Ionization and LC ESI Infrared Multiphoton Dissociation Fourier Transform Ion Cyclotron Resonance Mass Spectrometry. Anal. Chem. 1999, 71, 4397-4402.

13. Hofstadler, S. A.; Sannes-Lowery, K. A.; Griffey, R. H. Infrared Multiphoton Dissociation in an External Ion Reservoir. Anal. Chem. 1999, 71, 2067-2070.

14. Flora, J. W.; Muddiman, D. C. Selective, Sensitive, and Rapid Phosphopeptide Identification in Enzymatic Digests Using ESI-FTICR-MS with Infrared Multiphoton Dissociation. Anal. Chem. 2001, 73, 33053311.

15. Flora, J. W.; Muddiman, D. C. Gas-Phase Ion Unimolecular Dissociation for Rapid Phosphopeptide Mapping by IRMPD in a Penning Ion Trap: An Energetically Favored Process. J. Am. Chem. Soc. 2002, 124, 6546-6547.

16. Drader, J. J.; Hannis, J. C.; Hofstadler, S. A. Infrared Multiphoton Dissociation with a Hollow Fiber Waveguide. Anal. Chem. 2003, 75, 3669-3674.
17. Hofstadler, S. A.; Drader, J. J.; Gaus, H.; Hannis, J. C.; Sannes-Lowery, K. A. Alternative Approaches to Infrared Multiphoton Dissociation in an External Ion Reservoir. J. Am. Soc. Mass Spectrom. 2003, 14, 1413-1423.

18. Chalmers, M. J.; Quinn, J. P.; Blakney, G. T.; Emmett, M. R.; Mischak, H. Gaskell, S. J.; Marshall, A. G. Liquid Chromatography-Fourier Transform Ion Cyclotron Resonance Mass Spectrometric Characterization of Protein Kinase C Phosphorylation. J. Proteome Res. 2003, 2, 373-382.

19. Flora, J. W.; Muddiman, D. C. Determination of the Relative Energies of Activation for the Dissociation of Aromatic versus Aliphatic Phosphopeptides by ESI-FTICR-MS and IRMPD. J. Am. Soc. Mass Spectrom. 2004, 15, 121-127.

20. Chalmers, M. J.; Hakansson, K.; Johnson, R.; Smith, R.; Shen, J.; Emmett M. R.; Marshall, A. G. Protein Kinase A Phosphorylation Characterized by Tandem Fourier Transform Ion Cyclotron Resonance Mass Spectrometry. Proteomics 2004, 4, 970-981.

21. Tsybin, Y. O.; Ramstroem, M.; Witt, M.; Baykut, G.; Hakansson, P. Peptide and Protein Characterization by High-Rate Electron Capture Dissociation Fourier Transform Ion Cyclotron Resonance Mass Spectrometry. J. Mass Spectrom. 2004, 39, 719-729.

22. Yamada, N.; Suzuki, E.-I.; Hirayama, K. Effective Novel Dissociation Methods for Intact Protein: Heat-Assisted Nozzle-Skimmer Collisionally Induced Dissociation and Infrared Multiphoton Dissociation Using a Fourier Transform Ion Cyclotron Resonance Mass Spectrometer Equipped with a Micrometal Electrospray Ionization Emitter. Anal. Biochem. 2006, 348, 139-147.

23. Mihalca, R.; van der Burgt, Y. E. M.; McDonnell, L. A.; Duursma, M. Cerjak, I.; Heck, A. J. R.; Heeren, R. M. A. Combined Infrared Multiphoton Dissociation and Electron-Capture Dissociation Using Co-Linear and Overlapping Beams in Fourier Transform Ion Cyclotron Resonance Mass Spectrometry. Rapid Commun. Mass Spectrom. 2006, 20, 1838-1844.

24. Clowers, B. H.; Dodds, E. D.; Seipert, R. R.; Lebrilla, C. B. Site Determination of Protein Glycosylation Based on Digestion with Immobilized Nonspecific Proteases and Fourier Transform Ion Cyclotron Resonance Mass Spectrometry. J. Proteome Res. 2007, 6, 4032-4040.

25. Payne, A. H.; Glish, G. L. Thermally Assisted Infrared Multiphoton Photodissociation in a Quadrupole Ion Trap. Anal. Chem. 2001, 73, 3542-3548.

26. Steen, H.; Fernandez, M.; Ghaffari, S.; Pandey, A.; Mann, M. Phosphotyrosine Mapping in $\mathrm{Bcr} / \mathrm{Abl}$ Oncoprotein Using PhosphotyrosineSpecific Immonium Ion Scanning. Mol. Cell Proteomics 2003, 2, 138-145.

27. Trelle, M. B.; Jensen, O. N. Utility of Immonium Ions for Assignment of e-N-Acetyllysine-Containing Peptides by Tandem Mass Spectrometry. Anal. Chem. 2008, 80, 3422-3430.

28. Couttas, T. A.; Raftery, M. J.; Bernardini, G.; Wilkins, M. R. Immonium Ion Scanning for the Discovery of Post-Translational Modifications and Its Application to Histones. J. Proteome Res. 2008, 7, 2632-2641.

29. Crowe, M. C.; Brodbelt, J. S. Infrared Multiphoton Dissociation (IRMPD) and Collisionally Activated Dissociation of Peptides in a Quadrupole Ion Trap with Selective IRMPD of Phosphopeptides. J. Am. Soc. Mass Spectrom. 2004, 15, 1581-1592.

30. Wilson, J. J.; Brodbelt, J. S. Infrared Multiphoton Dissociation for Enhanced De Novo Sequence Interpretation of N-Terminal Sulfonated Peptides in a Quadrupole Ion Trap. Anal. Chem. 2006, 78, 6855-6862.

31. Pikulski, M.; Wilson, J. J.; Aguilar, A.; Brodbelt, J. S. Amplification of Infrared Multiphoton Dissociation Efficiency in a Quadruple Ion Trap Using IR-Active Ligands. Anal. Chem. 2006, 78, 8512-8517.

32. Pikulski, M.; Hargrove, A.; Shabbir, S. H.; Anslyn, E. V.; Brodbelt, J. S. Sequencing and Characterization of Oligosaccharides Using Infrared Multiphoton Dissociation and Boronic Acid Derivatization in a Quadrupole Ion Trap. J. Am. Soc. Mass Spectrom. 2007, 18, 2094-2106.

33. Gardner, M. W.; Vasicek, L. A.; Shabbir, S.; Anslyn, E. V.; Brodbelt, J. S. Chromogenic Cross-Linker for the Characterization of Protein Structure by Infrared Multiphoton Dissociation Mass Spectrometry. Anal. Chem. 2008, 80, 4807-4819.

34. Wysocki, V. H.; Tsaprailis, G.; Smith, L. L.; Breci, L. A. Mobile and Localized Protons: A Framework for Understanding Peptide Dissociation. J. Mass Spectrom. 2000, 35, 1399-1406.

35. Iavarone, A. T.; Jurchen, J. C.; Williams, E. R. Effects of Solvent on the Maximum Charge State and Charge State Distribution of Protein Ions Produced by Electrospray Ionization. J. Am. Soc. Mass Spectrom. 2000, 11, 976-985.

36. Iavarone, A. T.; Jurchen, J. C.; Williams, E. R. Supercharged Protein and Peptide Ions Formed by Electrospray Ionization. Anal. Chem. 2001, 73, $1455-1460$.

37. Iavarone, A. T.; Williams, E. R. Supercharging in Electrospray Ionization: Effects on Signal and Charge. Int. J. Mass Spectrom. 2002, 219, 63-72.

38. Iavarone, A. T.; Williams, E. R. Collisionally Activated Dissociation of Supercharged Proteins Formed by Electrospray Ionization. Anal. Chem. 2003, 75, 4525-4533.

39. Iavarone, A. T.; Williams, E. R. Mechanism of Charging and Supercharging Molecules in Electrospray Ionization. J. Am. Chem. Soc. 2003, 125, 2319-2327.

40. Black, D. M.; Payne, A. H.; Glish, G. L. Determination of Cooling Rates in a Quadrupole Ion Trap. J. Am. Soc. Mass Spectrom. 2006, 17, 932-938.

41. Schwartz, J. C. High-Q pulsed fragmentation in ion traps. U.S. Patent 7102 129, 2006. 